\title{
Oscillation criteria for fractional differential equations with mixed nonlinearities
}

\author{
Jing Shao ${ }^{1,2^{*}}$, Zhaowen Zheng $^{1}$ and Fanwei Meng ${ }^{1}$
}

"Correspondence:

shaojing99500@163.com

'School of Mathematical Sciences,

Qufu Normal University, Qufu,

Shandong 273165, P.R. China

${ }^{2}$ Department of Mathematics,

Jining University, Qufu, Shandong

273155, P.R. China

\begin{abstract}
Using the integral transformation and inequalities technique, new oscillation criteria are established for fractional differential equations with mixed nonlinearities involving Riemann-Liouville and Caputo fractional derivatives, which generalize and improve some recent results in literature.
\end{abstract}

MSC: 34A08; 34C10

Keywords: oscillation; fractional differential equation; Riemann-Liouville; Caputo; mixed nonlinearity

\section{Introduction}

Fractional differential equations appear more and more frequently in various research areas, such as in modeling mechanical and electrical properties of real materials, as well as in rheological theory and other physical problems, etc.; see, e.g., [1-6]. Differential equations involving the Riemann-Liouville, Caputo, and Grünwald-Letnikov differential operators of fractional order $0<q<1$ appear to be important in a number of works, especially in the theory of viscoelasticity and in hereditary solid mechanics.

In [3], the authors obtained new oscillation criteria for a fractional differential equations of the form

$$
{ }_{a} \mathbf{D}_{t}^{q} x+f_{1}(t, x)=v(t)+f_{2}(t, x), \quad \lim _{t \rightarrow a^{+}} J_{a}^{1-q} x(t)=a_{1},
$$

where the functions $f_{1}, f_{2}$, and $v$ are continuous.

In this paper, we consider the oscillation theory for a fractional differential equation with mixed nonlinearities of the type

$$
{ }_{a} \mathbf{D}_{t}^{q} x-p(t) x(t)+\sum_{i=1}^{m} q_{i}(t)|x(t)|^{\lambda_{i}-1} x(t)=v(t), \quad \lim _{t \rightarrow a^{+}} J_{a}^{1-q} x(t)=a_{1},
$$

where $\{p(t)\},\{v(t)\}$, and $\left\{q_{i}(t)\right\}(1 \leq i \leq m)$ are continuous functions on $[a,+\infty)$, and $\lambda_{i}$ $(1 \leq i \leq m)$ are ratios of odd positive integers with $\lambda_{1}>\cdots>\lambda_{l}>1>\lambda_{l+1}>\cdots>\lambda_{m}$.

By a solution of equation (2) we mean a function $x(t)$ which is defined for $t \geq a$ and satisfies equation (2). Such a solution is said to be oscillatory if it has arbitrarily large zeros on $[a, \infty)$; otherwise, it is called nonoscillatory. Equation (2) is said to be oscillatory if all its solutions are oscillatory.

C2013 Shao et al.; licensee Springer. This is an Open Access article distributed under the terms of the Creative Commons Attribution License (http://creativecommons.org/licenses/by/2.0), which permits unrestricted use, distribution, and reproduction in any medium, provided the original work is properly cited. 
By ${ }_{a} \mathbf{D}_{t}^{q}$ we denote the Riemann-Liouville differential operator of order $q$ with $0<q \leq 1$. For $p \geq 0$, the operator $J_{a}^{p}$ defined by

$$
J_{a}^{p} x(t)=\frac{1}{\Gamma(p)} \int_{a}^{t}(t-s)^{p-1} x(s) d s, \quad J_{a}^{0} x=x
$$

is called the Riemann-Liouville fractional integral operator. The Riemann-Liouville differential operator ${ }_{a} \mathbf{D}_{t}^{q}$ of order $q$ for $0<q \leq 1$ is defined by ${ }_{a} \mathbf{D}_{t}^{q} x(t)=\frac{d}{d t} J_{a}^{1-q} x(t)$ and, more generally, if $m \geq 1$ is an integer and $m-1<q \leq m$, then

$$
{ }_{a} \mathbf{D}_{t}^{q} x(t)=\frac{d^{m}}{d t^{m}} J_{a}^{m-q} x(t) .
$$

In [2, Lemma 5.3], under much weaker assumptions on $p(t), v(t)$ and $q_{i}(t)$, the initial value problem (2) is equivalent to the Volterra fractional integral equation

$$
\begin{aligned}
x(t)= & \frac{a_{1}(t-a)^{q-1}}{\Gamma(q)} \\
& +\frac{1}{\Gamma(q)} \int_{a}^{t}(t-s)^{q-1}\left[v(s)+p(s) x(s)-\sum_{i=1}^{m} q_{i}(s)|x(s)|^{\lambda_{i}-1} x(s)\right] d s .
\end{aligned}
$$

Therefore, a function $x(t)$ is a solution of (5) if and only if it is a solution of fractional differential equation (2).

In this paper, using the similar methods as that in [7], we give new oscillation criteria for equation (2) which generalize and improve the main results in paper [3] and references cited therein. Examples are given to each of these equations.

\section{Oscillation criteria of Riemann-Liouville fractional differential equations}

Lemma 2.1 (see [8]) Suppose that $X, Y$ and $U, V$ are nonnegative, then

(I) $\lambda X Y^{\lambda-1}-X^{\lambda} \leq(\lambda-1) Y^{\lambda}, \quad \lambda>1$,

(II) $\mu U V^{\mu-1}-U^{\mu} \geq(\mu-1) V^{\mu}, \quad 0<\mu<1$,

where each equality holds if and only if $X=Y$ or $U=V$.

Using the knowledge of linear algebra, we can easily obtain Lemma 2.2 .

Lemma 2.2 Let $\left(\alpha_{1}, \alpha_{2}, \ldots, \alpha_{m}\right)$ be an m-tuple satisfying $\alpha_{1}>\cdots>\alpha_{l}>1>\alpha_{l+1}>\cdots>\alpha_{m}>$ 0 . Then there exists an m-tuple $\left(\eta_{1}, \eta_{2}, \ldots, \eta_{m}\right)$ satisfying

$$
\sum_{i=1}^{l} \alpha_{i} \eta_{i}=\sum_{i=l+1}^{m} \alpha_{i} \eta_{i}
$$

with $\sum_{i=1}^{m} \eta_{i}=1$ and $0<\eta_{i}<1$ for $i=1,2, \ldots, m$.

Theorem 2.1 Assume

$$
p(t)>0, \quad q_{i}(t) \begin{cases}\geq 0 & \text { for } 1 \leq i \leq l \\ \leq 0 & \text { for } l+1 \leq i \leq m .\end{cases}
$$


Iffor some constant $K>0$,

$$
\liminf _{t \rightarrow \infty} t^{1-q} \int_{a}^{t}(t-s)^{q-1}\left(v(s)+K \sum_{i=1}^{m} p^{\frac{\lambda_{i}}{\lambda_{i}-1}}(s)\left|q_{i}(s)\right|^{\frac{1}{1-\lambda_{i}}}\right) d s=-\infty
$$

and

$$
\limsup _{t \rightarrow \infty} t^{1-q} \int_{a}^{t}(t-s)^{q-1}\left(v(s)+K \sum_{i=1}^{m} p^{\frac{\lambda_{i}}{\lambda_{i}-1}}(s)\left|q_{i}(s)\right|^{\frac{1}{1-\lambda_{i}}}\right) d s=\infty
$$

then every solution of equation (2) is oscillatory.

Proof Suppose to the contrary that there exists a nonoscillatory solution $x(t)$ of equation (2). Without loss of generality, we may suppose that $x(t)>0$ for $t \geq T$. It follows from equation (5) that

$$
\begin{aligned}
x(t) \leq & \frac{(t-a)^{q-1}}{\Gamma(q)}\left|a_{1}\right|+\frac{1}{\Gamma(q)} \int_{a}^{T}(t-s)^{q-1}|F(s)| d s \\
& +\frac{1}{\Gamma(q)} \int_{T}^{t}(t-s)^{q-1} v(s) d s \\
& +\frac{1}{\Gamma(q)} \int_{T}^{t}(t-s)^{q-1}\left(p(s) x(s)-\sum_{i=1}^{m} q_{i}(s) x^{\lambda_{i}}(s)\right) d s
\end{aligned}
$$

where $F(s)=v(s)+p(s) x(s)-\sum_{i=1}^{m} q_{i}(s) x^{\lambda_{i}}(s)$.

For $t \geq T$, multiplying the inequality (11) by $\Gamma(q) t^{1-q}$, we find that

$$
\begin{aligned}
\Gamma(q) t^{1-q} x(t) \leq & C(T)+t^{1-q} \int_{T}^{t}(t-s)^{q-1} v(s) d s \\
& +t^{1-q} \int_{T}^{t}(t-s)^{q-1}\left[\sum_{i=1}^{l}\left(\lambda_{i} p(s) x(s)-q_{i}(s) x^{\lambda_{i}}(s)\right)\right] d s \\
& +t^{1-q} \int_{T}^{t}(t-s)^{q-1}\left[\sum_{i=l+1}^{m}\left(-A p(s) x(s)+\left|q_{i}(s)\right| x^{\lambda_{i}}(s)\right)\right] d s,
\end{aligned}
$$

where $C(T)=\left(\frac{T}{T-a}\right)^{1-q}\left|a_{1}\right|+\int_{a}^{T}\left(\frac{T}{T-s}\right)^{1-q}|F(s)| d s$ and $A=\left(\sum_{i=1}^{l} \lambda_{i}-1\right) /(m-l)>0$.

For $t \geq T$, set

$$
\begin{aligned}
& X_{i}=q_{i}^{\frac{1}{\lambda_{i}}}(s) x(s) \quad \text { and } \quad Y_{i}=\left(p(s) q_{i}^{-\frac{1}{\lambda_{i}}}(s)\right)^{\frac{1}{\lambda_{i}-1}}, \quad 1 \leq i \leq l, \\
& U_{i}=\left|q_{i}(s)\right|^{\frac{1}{\lambda_{i}}} x(s) \text { and } \quad V_{i}=\left(\frac{A}{\lambda_{i}} p(s)\left|q_{i}(s)\right|^{-\frac{1}{\lambda_{i}}}\right)^{\frac{1}{\lambda_{i}-1}}, \quad l+1 \leq i \leq m,
\end{aligned}
$$


using Lemma 2.1(I) for $1 \leq i \leq l$ and (II) for $l+1 \leq i \leq m$ to obtain

$$
\begin{aligned}
\Gamma(q) t^{1-q} x(t) \leq & C(T)+t^{1-q} \int_{T}^{t}(t-s)^{q-1} v(s) d s \\
& +t^{1-q} \int_{T}^{t}(t-s)^{q-1} \sum_{i=1}^{l}\left(\lambda_{i}-1\right) p^{\frac{\lambda_{i}}{\lambda^{-1}}}(s) q_{i}^{\frac{1}{1-\lambda_{i}}}(s) d s \\
& +t^{1-q} \int_{T}^{t}(t-s)^{q-1} \sum_{i=l+1}^{m}\left(1-\lambda_{i}\right)\left(\frac{\lambda_{i}}{A}\right)^{\frac{\lambda_{i}}{1-\lambda_{i}}} p^{\frac{\lambda_{i}}{\lambda_{i}-1}}(s)\left|q_{i}(s)\right|^{\frac{1}{1-\lambda_{i}}} d s \\
\leq & C(T)+t^{1-q} \int_{T}^{t}(t-s)^{q-1} v(s) d s \\
& +t^{1-q} \int_{T}^{t}(t-s)^{q-1} K \sum_{i=1}^{m} p^{\frac{\lambda_{i}}{\lambda_{i}-1}}(s)\left|q_{i}(s)\right|^{\frac{1}{1-\lambda_{i}}} d s, \quad t \geq T,
\end{aligned}
$$

where $K=\max \left\{\lambda_{1}-1, \max _{l+1 \leq i \leq m}\left(1-\lambda_{i}\right)\left(\frac{\lambda_{i}}{A}\right)^{\frac{\lambda_{i}}{1-\lambda_{i}}}\right\}$. Note that the improper integral on the right is divergent. Taking the limit inferior of both sides of inequality (13) as $t \rightarrow \infty$, we get a contradiction to condition (9). In the case $x(t)$ is eventually negative, a similar argument leads to a contradiction to (10). This completes the proof of Theorem 2.1.

Following the proof of Theorem 2.1, we can easily obtain the following corollaries.

Letting $l=m$ in equation (2), we get $\lambda_{1}>\lambda_{2}>\cdots>\lambda_{m}>1$.

Corollary 2.1 Suppose $p(t)>0, q_{i}(t) \geq 0,1 \leq i \leq m$. If (9), (10) hold for some constant $K_{1}>0$, then equation (2) is oscillatory.

Proof Suppose to the contrary that there exists a nonoscillatory solution $x(t)$ of equation (2). Without loss of generality, we may suppose that $x(t)$ is an ultimately positive solution of equation (2). So, there exists $T>a$ such that $x(t)>0$ for $t \geq T$. It follows from equation (2) that

$$
\begin{aligned}
\Gamma(q) t^{1-q} x(t) \leq & C(T)+t^{1-q} \int_{T}^{t}(t-s)^{q-1} v(s) d s \\
& +t^{1-q} \int_{T}^{t}(t-s)^{q-1}\left[\sum_{i=1}^{m}\left(\frac{1}{m} p(s) x(s)-q_{i}(s) x^{\lambda_{i}}(s)\right)\right] d s .
\end{aligned}
$$

For $t \geq T$, set

$$
X_{i}=q_{i}^{\frac{1}{\lambda_{i}}}(s) x(s) \quad \text { and } \quad Y_{i}=\left(\frac{1}{m \lambda_{i}} p(s) q_{i}^{-\frac{1}{\lambda_{i}}}(s)\right)^{\frac{1}{\lambda_{i}-1}}, \quad 1 \leq i \leq m
$$

and, using Lemma 2.1(I), we obtain

$$
\begin{aligned}
\Gamma(q) t^{1-q} x(t) \leq & C(T)+t^{1-q} \int_{T}^{t}(t-s)^{q-1} v(s) d s \\
& +t^{1-q} \int_{T}^{t}(t-s)^{q-1} K_{1} \sum_{i=1}^{m} p^{\frac{\lambda_{i}}{\lambda_{i}-1}}(s)\left|q_{i}(s)\right|^{\frac{1}{1-\lambda_{i}}} d s, \quad t \geq T
\end{aligned}
$$


where $K_{1} \geq \frac{\lambda_{1}-1}{m}$. The remaining part is similar to that of Theorem 2.1, so we omit the details. The proof of Corollary 2.1 is finished.

If $l=0$ in equation (2), then $1>\lambda_{1}>\lambda_{2}>\cdots>\lambda_{m}$. Similarly, we obtain the following corollary.

Corollary 2.2 Suppose $p(t)<0, q_{i}(t) \leq 0,1 \leq i \leq m$. If (9), (10) hold for some constant $K_{2}>0$, then equation (2) is oscillatory.

If $p(s) \equiv 0$ and $1<l<m$ in equation (2), we obtain the following corollary.

Corollary 2.3 Assume

$$
q_{i}(t) \begin{cases}\geq 0 & \text { for } 1 \leq i \leq l \\ \leq 0 & \text { for } l+1 \leq i \leq m\end{cases}
$$

If there exists a positive function $r(t)$ on $[a, \infty)$ such that for some constant $K_{3}>0$,

$$
\liminf _{t \rightarrow \infty} t^{1-q} \int_{a}^{t}(t-s)^{q-1}\left(v(s)+K_{3} \sum_{i=1}^{m} r^{\frac{\lambda_{i}}{\lambda_{i}-1}}(s)\left|q_{i}(s)\right|^{\frac{1}{1-\lambda_{i}}}\right) d s=-\infty
$$

and

$$
\limsup _{t \rightarrow \infty} t^{1-q} \int_{a}^{t}(t-s)^{q-1}\left(v(s)+K_{3} \sum_{i=1}^{m} r^{\frac{\lambda_{i}}{\lambda_{i}-1}}(s)\left|q_{i}(s)\right|^{\frac{1}{1-\lambda_{i}}}\right) d s=\infty
$$

then every solution of equation (2) is oscillatory.

Proof For $\lambda_{1}>\cdots>\lambda_{l}>1>\lambda_{l+1}>\cdots>\lambda_{m}$, by Lemma 2.2, there exists an $m$-tuple $\left(\eta_{1}, \ldots, \eta_{m}\right)$ satisfying

$$
\sum_{i=1}^{l} \lambda_{i} \eta_{i}=\sum_{i=l+1}^{m} \lambda_{i} \eta_{i}
$$

Suppose to the contrary that there exists a nonoscillatory positive solution $x(t)$ for $t \geq T$. It follows from equation (2) that

$$
\begin{aligned}
\Gamma(q) t^{1-q} x(t) \leq & C(T)+t^{1-q} \int_{T}^{t}(t-s)^{q-1} v(s) d s \\
& +t^{1-q} \int_{T}^{t}(t-s)^{q-1}\left[\sum_{i=1}^{l}\left(\lambda_{i} \eta_{i} r(s) x(s)-q_{i}(s) x^{\lambda_{i}}(s)\right)\right] d s \\
& +t^{1-q} \int_{T}^{t}(t-s)^{q-1}\left[\sum_{i=l+1}^{m}\left(-\lambda_{i} \eta_{i} r(s) x(s)+\left|q_{i}(s)\right| x^{\lambda_{i}}(s)\right)\right] d s .
\end{aligned}
$$

The remainder of the proof is similar, so we omit the details. 
Remark 2.1 When $l=2, m=2$ and $p(t) \equiv 0$, a similar result is obtained by [3]. However, our result, Corollary 2.3, is different from that obtained in [3] since an auxiliary function $r(t)$ is involved.

Remark 2.2 The results remain valid for fractional differential equations involving the Riemann-Liouville differential operator ${ }_{a} \mathbf{D}_{t}^{q}$ of order $q$ with $m-1<q \leq m$, where $m \geq 1$ is an integer of the form

$$
\begin{aligned}
& { }_{a} \mathbf{D}_{t}^{q} x-p(t) x(t)+\sum_{i=1}^{m} q_{i}(t)|x(t)|^{\lambda_{i}-1} x(t)=v(t), \\
& { }_{a} \mathbf{D}_{t}^{q-k} x(a)=a_{k}, \quad k=1, \ldots, m-1, \\
& \lim _{t \rightarrow a^{+}} J_{a}^{m-q} x(t)=a_{m} .
\end{aligned}
$$

In fact, initial value problem (15) is equivalent to the Volterra fractional integral equation

$$
x(t)=\sum_{k=1}^{m} \frac{a_{k}(t-a)^{q-k}}{\Gamma(q-k+1)}+\frac{1}{\Gamma(q)} \int_{a}^{t}(t-s)^{q-1}\left[v(s)+p(s) x(s)-\sum_{i=1}^{m} q_{i}(s)|x(s)|^{\lambda_{i}-1} x(s)\right] d s .
$$

We have similar theorems in such a case as that in $0<q \leq 1$.

\section{Oscillation of Caputo fractional differential equations}

In this section, we give oscillation criteria for equation (2) under the Caputo fractional derivatives approach. Caputo's definition can be written as

$$
{ }_{a}^{C} D_{t}^{q} x(t)=J_{a}^{m-q} x^{(m)}(t), \quad m-1<q \leq m,
$$

where $x(t)$ is an $m$ times differentiable function. The initial value problem of equation (2) should be replaced by

$$
{ }_{a}^{C} D_{t}^{q} x-p(t) x(t)+\sum_{i=1}^{m} q_{i}(t)|x(t)|^{\lambda_{i}-1} x(t)=v(t), \quad x^{(k)}(a)=a_{k}, \quad k=0,1, \ldots, m .
$$

Moreover, the corresponding Volterra fractional integral equation, see [9, Lemma 6.2], becomes

$$
\begin{aligned}
x(t)= & \sum_{k=0}^{m-1} \frac{a_{k}(t-a)^{k}}{k !} \\
& +\frac{1}{\Gamma(q)} \int_{a}^{t}(t-s)^{q-1}\left[v(s)+p(s) x(s)-\sum_{i=1}^{m} q_{i}(t)|x(t)|^{\lambda_{i}-1} x(t)\right] d s .
\end{aligned}
$$

Using similar methods, the oscillation criteria can be obtained for Caputo's case.

Theorem 3.1 Assume that condition (8) holds. If

$$
\liminf _{t \rightarrow \infty} t^{1-m} \int_{a}^{t}(t-s)^{q-1}\left(v(s)+K \sum_{i=1}^{m} p^{\frac{\lambda_{i}}{\lambda_{i}-1}}(s)\left|q_{i}(s)\right|^{\frac{1}{1-\lambda_{i}}}\right) d s=-\infty
$$


and

$$
\limsup _{t \rightarrow \infty} t^{1-m} \int_{a}^{t}(t-s)^{q-1}\left(v(s)+K \sum_{i=1}^{m} p^{\frac{\lambda_{i}}{\lambda_{i}-1}}(s)\left|q_{i}(s)\right|^{\frac{1}{1-\lambda_{i}}}\right) d s=\infty
$$

for some constant $K>0$, then every solution of equation (16) is oscillatory.

Corollary 3.1 Suppose $p(t)>0, q_{i}(t) \geq 0,1 \leq i \leq m$. If (17), (18) hold for some constant $K_{1}>0$, then equation (16) is oscillatory.

Corollary 3.2 Suppose $p(t)>0, q_{i}(t) \leq 0,1 \leq i \leq m$. If (17), (18) hold for some constant $K_{2}>0$, then equation (16) is oscillatory.

Corollary 3.3 If condition (14) holds, and there exists a positive function $r(t)$ on $[a, \infty)$ such that

$$
\liminf _{t \rightarrow \infty} t^{1-m} \int_{a}^{t}(t-s)^{q-1}\left[v(s)+K_{3} \sum_{i=1}^{m} r^{\frac{\lambda_{i}}{\lambda_{i}-1}}(s)\left|q_{i}(s)\right|^{\frac{1}{1-\lambda_{i}}}\right] d s=-\infty
$$

and

$$
\limsup _{t \rightarrow \infty} t^{1-m} \int_{a}^{t}(t-s)^{q-1}\left[v(s)+K_{3} \sum_{i=1}^{m} r^{\frac{\lambda_{i}}{\lambda_{i}-1}}(s)\left|q_{i}(s)\right|^{\frac{1}{1-\lambda_{i}}}\right] d s=\infty
$$

for some constant $K_{3}>0$, then every solution of equation (16) is oscillatory.

Remark 3.1 In [1], the Grünwald-Letnikov fractional derivative, under the assumption that the function $x(t)$ must be $m+1$ times continuously differentiable, can be obtained from (4) under the same assumption by performing repeatedly integration by parts and differentiation. Therefore, our results are suitable for the Grünwald-Letnikov fractional derivative approaches, too.

\section{Examples}

In this section, we give the following examples to illustrate the effectiveness of our theorems.

Example 4.1 Consider the following fractional differential equation:

$$
{ }_{0} \mathbf{D}_{t}^{\frac{1}{2}} x-t^{2} x+2 t^{3}|x|^{\frac{1}{2}} x-t|x|^{-\frac{1}{2}} x=\sin t, \quad \lim _{t \rightarrow 0^{+}} J_{0}^{\frac{1}{2}} x(t)=0 .
$$

It is easy to obtain $K=\frac{1}{2}$. Using Theorem 2.1, we get

$$
\begin{aligned}
& \liminf _{t \rightarrow \infty} t^{1-q} \int_{a}^{t}(t-s)^{q-1}\left(v(s)+K \sum_{i=1}^{m} p^{\frac{\lambda_{i}}{\lambda_{i}-1}}(s)\left|q_{i}(s)\right|^{\frac{1}{1-\lambda_{i}}}\right) d s \\
& \quad=\liminf _{t \rightarrow \infty} t^{\frac{1}{2}} \int_{0}^{t}(t-s)^{-\frac{1}{2}}\left(\sin s+\frac{5}{8}\right) d s=\liminf _{t \rightarrow \infty} t^{\frac{1}{2}}\left(\int_{0}^{t}(t-s)^{-\frac{1}{2}} \sin s d s+\frac{5}{4} t^{\frac{1}{2}}\right) .
\end{aligned}
$$


Since the integral $\int_{0}^{t}(t-s)^{-\frac{1}{2}} \sin s d s$ is negative for $t=2 k \pi-\frac{\pi}{2}, k=0,1,2, \ldots$, we get

$$
\liminf _{t \rightarrow \infty} t^{\frac{1}{2}}\left(\int_{0}^{t}(t-s)^{-\frac{1}{2}} \sin s d s+\frac{5}{4} t^{\frac{1}{2}}\right)=-\infty
$$

Furthermore, for the same reason,

$$
\limsup _{t \rightarrow \infty} t^{\frac{1}{2}}\left(\int_{0}^{t}(t-s)^{-\frac{1}{2}} \sin s d s+\frac{5}{4} t^{\frac{1}{2}}\right)=\infty
$$

So, equation (19) is oscillatory.

Example 4.2 Consider the following fractional differential equation:

$$
{ }_{0} \mathbf{D}_{t}^{1 / 2} x-t^{2} x+2 t^{3}|x|^{\frac{1}{2}} x=\frac{t^{1-q}}{\Gamma(2-q)}+2 t^{9 / 2}-t^{3}, \quad \lim _{t \rightarrow 0^{+}} J_{0}^{1 / 2} x(t)=0
$$

Since $\frac{t^{1-q}}{\Gamma(2-q)}+2 t^{9 / 2}-t^{3} \geq 0$ and

$$
\begin{aligned}
& t^{1-q} \int_{a}^{t}(t-s)^{q-1}\left(v(s)+K \sum_{i=1}^{m} p^{\frac{\lambda_{i}}{\lambda_{i}-1}}(s)\left|q_{i}(s)\right|^{\frac{1}{1-\lambda_{i}}}\right) d s \\
& \geq t^{1-q} \int_{a}^{t}(t-s)^{q-1} K \sum_{i=1}^{m} p^{\frac{\lambda_{i}}{\lambda_{i}-1}}(s) \mid q_{i}(s)^{\frac{1}{1-\lambda_{i}}} d s \\
& =t^{1 / 2} \int_{0}^{t} \frac{K}{4}(t-s)^{-1 / 2} d s \\
& =\frac{K}{2} t
\end{aligned}
$$

we get that neither (9) nor (10) is satisfied. We can also easily verify that $x(t)=t$ is a nonoscillatory solution of (20).

\section{Competing interests}

The authors declare that they have no competing interests.

\section{Authors' contributions}

JS completed the main part of this article, ZZ and FM corrected the main theorems. All authors read and approved the final manuscript.

\section{Acknowledgements}

The authors thank the referee for his/her valuable suggestions to improve the present article. This project is supported by the NSF of China (Grants 11171178 and 11271225), Science and Technology Project of High Schools of Shandong Province (Grant J12LI52) and Program for Scientific Research Innovation Team in Colleges and Universities of Shandong Province.

Received: 26 May 2013 Accepted: 24 October 2013 Published: 18 Nov 2013

\section{References}

1. Podlubny, I: Fractional Differential Equations. Academic Press, San Diego (1999)

2. Diethelm, K: The Analysis of Fractional Differential Equations. Springer, Berlin (2010)

3. Grace, SR, Agarwal, RP, Wang, PJY, Zafer, A: On the oscillation of fractional differential equations. Fract. Calc. Appl. Anal. 15(2), 222-231 (2012)

4. Denton, Z, Vatsala, AS: Fractional integral inequalities and applications. Comput. Math. Appl. 59, 1087-1094 (2010)

5. Graef, JR, Kong, L, Yang, B: Positive solutions for a semipositone fractional boundary value problem with a forcing term. Fract. Calc. Appl. Anal. 15(1), 8-24 (2012) 
6. Samko, SG, Kilbas, AA, Marichev, OI: Fractional Integrals and Derivatives: Theory and Applications. Gordon \& Breach, Amsterdam (1993)

7. Zheng, Z, Wang, X, Han, H: Oscillation criteria for forced second order differential equations with mixed nonlinearities. Appl. Math. Lett. 22, 1096-1101 (2009)

8. Hardy, GH, Littlewood, JE, Polya, G: Inequalities, 2nd edn. Cambridge University Press, Cambridge (1988)

9. Bai, Y, Liu, L: New oscillation criteria for second-order delay differential equations with mixed nonlinearities. Discrete Dyn. Nat. Soc. 2010, Article ID 796256 (2010)

10.1186/1687-1847-2013-323

Cite this article as: Shao et al.: Oscillation criteria for fractional differential equations with mixed nonlinearities. Advances in Difference Equations 2013, 2013:323

Submit your manuscript to a SpringerOpen ${ }^{\circ}$ journal and benefit from:

- Convenient online submission

- Rigorous peer review

- Immediate publication on acceptance

- Open access: articles freely available online

- High visibility within the field

- Retaining the copyright to your article

Submit your next manuscript at $>$ springeropen.com 